\title{
Spectroscopy at the solar limb
}

\section{Average off-limb profiles and Doppler shifts of Ca II $H^{\star}, \star \star$}

\author{
C. A. R. Beck ${ }^{1,2}$ and R. Rezaei ${ }^{3}$ \\ ${ }^{1}$ Instituto de Astrofísica de Canarias, C/ Vía Lactéa s/n, 38205 La Laguna, Tenerife, Spain \\ e-mail: cbeck@iac.es \\ 2 Departamento de Astrofísica, Universidad de La Laguna, 38205 La Laguna, Tenerife, Spain \\ ${ }^{3}$ Kiepenheuer-Institut für Sonnenphysik, Schöneckstr. 6, 79104 Freiburg, Germany \\ e-mail: rrezaei@kis.uni-freiburg.de
}

Received 11 May 2010 / Accepted 5 May 2011

\section{ABSTRACT}

\begin{abstract}
Aims. We present constraints on the thermodynamical structure of the chromosphere from ground-based observations of the $\mathrm{Ca}$ II $\mathrm{H}$ line profile near and off the solar limb.

Methods. We obtained a slit-spectrograph data set of the $\mathrm{Ca}$ II $\mathrm{H}$ line with a high signal-to-noise ratio in a field of view extending $20^{\prime \prime}$ across the limb. We analyzed the spectra for the characteristic properties of average and individual off-limb spectra. We used various tracers of the Doppler shifts, such as the location of the absorption core, the ratio of the two emission peaks $\mathrm{H}_{2 \mathrm{~V}}$ and $\mathrm{H}_{2 \mathrm{R}}$, and intensity images at a fixed wavelength.

Results. The average off-limb profiles show a smooth variation with increasing limb distance. The line width increases up to a height of about $2 \mathrm{Mm}$ above the limb. The profile shape is fairly symmetric with nearly identical $\mathrm{H}_{2 \mathrm{~V}}$ and $\mathrm{H}_{2 \mathrm{R}}$ intensities; at a height of $5 \mathrm{Mm}$, it changes into a single Gaussian without emission peaks. We find that all off-limb spectra show large Doppler shifts that fluctuate on the smallest resolved spatial scales. The variation is more prominent in cuts parallel to the solar limb than on those perpendicular to it. As far as individual structures can be unequivocally identified at our spatial resolution, we find a specific relation between intensity enhancements and Doppler shifts: elongated brightenings are often flanked all along their extension by velocities in opposite directions.

Conclusions. The average off-limb spectra of $\mathrm{Ca}$ II $\mathrm{H}$ present a good opportunity to test static chromospheric atmosphere models because they lack the photospheric contribution that is present in disk-center spectra. We suggest that the observed relation between intensity enhancements and Doppler shifts could be caused by waves propagating along the surfaces of flux tubes: an intrinsic twist of the flux tubes or a wave propagation inclined to the tube axis would cause a helical shape of the Doppler excursions, visible as opposite velocity at the sides of the flux tube. Spectroscopic data allow one to distinguish this from a sausage-mode oscillation where the maximum Doppler shift and the tube axis would coincide.
\end{abstract}

Key words. Sun: chromosphere - techniques: spectroscopic - methods: data analysis

\section{Introduction}

The solar chromosphere is a very variable atmospheric layer governed by dynamical processes. Part of its organization is provided by the photospheric magnetic fields and their extension into the upper solar atmosphere, but the main characteristic of the chromosphere is its dynamical evolution. The chromospheric temperature is higher than that of the underlying photosphere, but because the gas is transparent owing to its low density, energy sources other than radiation have to maintain the pure existence of the chromosphere (see, e.g., Rutten 2010, and references therein). Different methods have been suggested to provide the necessary energy, ranging from a purely mechanical transfer of energy by propagating waves to various processes related to the magnetic field such as reconnection (Biermann 1948; Narain \& Ulmschneider 1996; Kalkofen 1996; Carlsson \& Stein 1997; Fossum \& Carlsson 2005; Socas-Navarro 2005).

\footnotetext{
* Appendices are available in electronic form at http://www . andaa.org

$\star \star$ The Data set is available in electronic form at the CDS via anonymous ftp to cdsarc.u-strasbg. fr (130.79.128.5) or via http://cdsarc.u-strasbg.fr/viz-bin/qcat?]/A+A/531/A173
}

Because of its low gas density, the assumption of local thermal equilibrium (LTE) is not justified in the chromosphere. Spectral lines like $\mathrm{H} \alpha$, Ca II $\mathrm{H}$, the Ca II IR triplet, or He I at $1083 \mathrm{~nm}$ contain the information on the thermodynamical and magnetic properties of the chromosphere (e.g., Balasubramaniam et al. 2004; Socas-Navarro \& Elmore 2005; Pietarila et al. 2007; Rezaei et al. 2007; Beck et al. 2008; Cauzzi et al. 2008; Centeno et al. 2010), but the non-LTE conditions of the line formation complicate the analysis of the spectra. Because there are relatively few approaches to directly derive the desired information from individual spectra, the investigation of the chromosphere is often made with average spectra of some sort. This allowed static atmospheric models corresponding to a temporally and spatially averaged chromosphere to be developed (Vernazza et al. 1981; Fontenla et al. 2006), but their relevance for individual spectra is under debate (Fontenla et al. 2007).

For these studies, usually spectra observed on the center of the solar disk were used. Because most of the chromospheric spectral lines have a significant contribution from photospheric layers in their line wings, disk-center spectra can be contaminated with photospheric information as well. Observations near 
and off the solar limb remove the photospheric contribution because the line of sight (LOS) for observations above the solar limb is not passing through photospheric layers anymore.

We aim to improve the observational constraints on the solar chromosphere by investigating medium-resolution $\mathrm{Ca}$ II $\mathrm{H}$ spectra near and above the limb. The data were obtained in 2009 at the German Vacuum Tower Telescope (VTT, Schröter et al. 1985), Izaña. Thanks to the Kiepenheuer Institute adaptive optics system (KAOS; von der Lühe et al. 2003), they are intermediate between the high-resolution imaging data in $\mathrm{Ca}$ II $\mathrm{H}$ from the HINODE satellite (Kosugi et al. 2007) and the spectroscopic data obtained before the advent of adaptive optics (AO) systems (Zirker 1962; Beckers 1968; Zirker 1968), providing a full linespectrum with high spectral resolution. The observations are described in Sect. 2. The data reduction is discussed in Sect. 3, whereas Sect. 4 displays our observational findings. Section 5 summarizes our results, which are discussed in Sect. 6. Section 7 presents our conclusions.

\section{Observations}

During an observation campaign for a study of the center-tolimb variation (CLV) of Ca II H spectra in August 2009, we obtained some data sets at the very limb as well. We selected the one with the best spatial resolution for the present study, taken on 25 Aug. 2009 between 08:43 and 09:15 UT. The spectra were taken with the POlarimetric LIttrow Spectrograph (POLIS, Beck et al. 2005b) at the VTT, a slit-spectrograph spectropolarimeter with two channels at $630 \mathrm{~nm}$ ("red channel") and in $\mathrm{Ca}$ II $\mathrm{H}$ at $396.85 \mathrm{~nm}$ ("blue channel"). The observed wavelength range of the red channel covered a range slightly more toward the blue than the standard configuration, containing the Fe I line at $630.15 \mathrm{~nm}$ and the $\mathrm{O}_{\mathrm{I}}$ line at $630.06 \mathrm{~nm}$ instead of the Fe I line at $630.25 \mathrm{~nm}$. Since the removal of the polarizing beamsplitter in the blue channel in 2005 to increase the low light level in the near-UV, the Ca channel provides spectroscopy only. We used an integration time of about 13 sec per scan step. The spectral sampling in the Ca channel was $1.95 \mathrm{pm}$ per pixel at a nominal resolution of the spectrograph of $\Delta \lambda / \lambda=220$, 000. The slit width corresponded to 0.5 , and the slit was moved in 134 steps of 0.3 across the solar image, yielding a field of view (FOV) of about $40^{\prime \prime} \times 70^{\prime \prime}$. The spatial sampling along the slit corresponded to about 0.3 . The center of the FOV was located on the solar disk at $\left(+37^{\prime \prime},+920^{\prime \prime}\right)$, giving a heliocentric angle of 74 degree. The slit was inclined by 30 deg to the solar limb and protruded at maximum about $20^{\prime \prime}$ over the limb in the Ca channel. The KAOS system was locked on a bright facula near the center of the FOV. Because at the VTT the scanning system of either the main spectrograph or POLIS is independent of the AO, the AO could stay locked on the same point of the solar surface except for one failure $\left(x \sim 23^{\prime \prime}\right.$, marked in Fig. 3 below) where the AO lock point jumped by about $2^{\prime \prime}$ to another near-by facula.

During the whole CLV campaign, we intermittently took data sets with the same integration time on the solar disk center in between the CLV observations. These disk center data sets allowed us to derive an intensity normalization curve with which we were able to normalize the intensity of each observation in (arbitrary) detector counts to the disk-center intensity at the time of the observations (cf., e.g., Zirker 1968). In addition to the regular observations, we also carried out several types of measurements to determine the stray-light level in the spectra. Here we will only discuss an observation where half of the FOV was blocked by a metal plate in the telescope focal plane up front of the AO. The data was used to derived an estimate of the off-limb stray light and the spatial point spread function (PSF) of POLIS. The measurements for determining other stray-light contributions and their evaluation are discussed in more detail in another publication (Beck et al. 2011).

We estimate the spatial resolution of the data to be about $1^{\prime \prime}-1.5^{\prime \prime}$, with a residual uncorrected image motion of less than 0.'5 (see Appendix A).

\section{Data reduction}

The data of both POLIS channels were reduced by the approaches described in Beck et al. (2005b,a). This includes the correction for dark current and the correction for flat-field imperfections, and in the case of the Ca channel a correction for the order-selecting pre-filter in front of the camera. To improve the data quality, we added some more reduction steps before we evaluated the spectra, however.

Stray-light correction. Because in the end we aim to arrive at a conversion from observed intensities in detector counts into the absolute energy of the radiation, the data needed to be corrected for stray-light contributions. In this context, we modeled the spectrum $I(\lambda)$ of a certain pixel $(x, y)$ in the FOV as

$I_{\text {obs }}(\lambda, x, y)=I_{\text {true }}(\lambda, x, y)+\alpha I_{\text {local }}(\lambda)+\beta\left\langle I_{\text {local }}\right\rangle$,

where $I_{\text {local }}(\lambda)$ is some average profile of the local FOV, and $\left\langle I_{\text {local }}\right\rangle$ an average intensity value without wavelength dependence. From the analysis of the stray-light measurements mentioned in the previous section, we derived typical values of $\beta=5 \%$ and $\alpha=10 \%$ as lower limit across the full FOV for the contributions of the parasitic ${ }^{1}$ and local stray light, respectively. The observed spectra located on the solar disk were thus corrected by subtracting $5 \%$ of the intensity near $396.4 \mathrm{~nm}$ in the average profile from the whole spectrum, and then $10 \%$ of the average spectrum $I_{\text {local }}(\lambda)$ was subtracted from each profile.

Off-limb correction. It became clear from the observed spectra where the slit crossed the limb, or from the measurement with the half-blocked FOV that the stray-light contribution $\alpha$ depends on the distance to the limb (or the blocking edge). To take this effect into account, we scaled $\alpha$ down with increasing limb distance by the spatial intensity variation that was observed with the half-blocked FOV (black line in Fig. 1). We cut the observed intensity curve at the approximate location of the edge and normalized the first point to $10 \%$ (value of $\alpha$ on the disk; see the inset). The reduced $\alpha$ was then used for all off-limb spectra as a function of their distance $d$ to the "white-light" limb seen at about $396.4 \mathrm{~nm}$.

It turned out that subtraction of $\alpha(d) I_{\text {local }}$ with $\alpha\left(d=0^{\prime \prime}\right)=$ $10 \%$ did not yield a good correction beyond the limb because the off-disk stray light was not fully compensated. We first tried to use an average disk-center profile with its higher intensity level instead of $I_{\text {local }}$, but this over-compensated the stray light and left strong residuals of the line blends in the Ca wing because they were located at slightly different wavelengths in the average disk center profile. We therefore decided to multiply the intensity of the average profile $I_{\text {local }}$ with a scaling coefficient, and by trialand-error obtained that an increase by a factor of 2 gave a satisfactory correction off the disk. We first attributed this to the fact

\footnotetext{
1 Spectrally un-dispersed light that still reaches the CCD cameras by scattering inside the instrument without passing through the grating.
} 


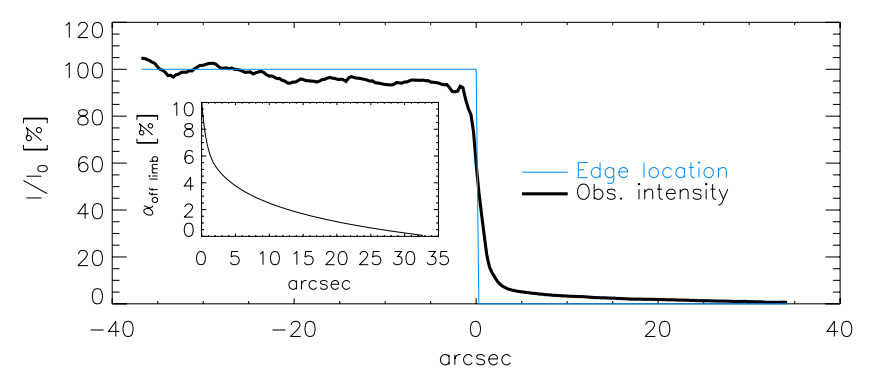

Fig. 1. Intensity variation along the slit near $396.4 \mathrm{~nm}$ for a partly blocked FOV. Thick black: observed intensity variation. Blue: location of the sharp edge. The inset shows the derived stray-light contribution $\alpha$ as a function of the limb distance.

that the scanned FOV is significantly smaller than the full FOV that passes through the field stop of POLIS, thus there is also an area with higher light level than near the limb. An in-depth study of the stray-light level (Beck et al. 2011) using a semi-empirical spatial PSF for POLIS later revealed that the stray light from the close surroundings of a given spatial point in the quiet Sun is indeed closer to $\alpha=20 \%$ than the $\alpha=10 \%$ used that was derived from profiles in the umbra of sunspots. The stray-light correction uses $\alpha I_{\text {local }}$ in Eq. (1), where the factor of 2 can therefore be attributed to either $\alpha$ or $I_{\text {local }}$. If one attributes it to $\alpha$ as suggested by the PSF, the effective stray-light correction consisted of a correction for $20 \%$ stray light on-disk using the average profile of the observation $I_{\text {local }}$, and for a stray-light coefficient $\alpha(d)$ beyond the limb corresponding to twice the values shown in the inset of Fig. 1.

The observation of the knife-edge function with the halfblocked focal plane only covers the optics downstream of the telescope focal plane. Thus neither the PSF of the telescope nor fluctuations of the scattering in the Earth's atmosphere are taken into account in the stray-light correction. Fluctuations of the scattering presumably were minor during the observations (see Fig. A.2). The stray-light correction is smooth across the limb location because $\alpha\left(d=0^{\prime \prime}\right)$ was normalized to the on-disk value, which still holds regardless if $\alpha$ or $I_{\text {local }}$ are scaled up, and because the doubled stray-light amount $\alpha I_{\text {local }}$ with $\alpha=20 \%$ was subtracted on the disk.

This approach for the off-limb correction yielded a residual intensity of below about $0.2 \%$ of $I_{\mathrm{c}}$ for all off-limb spectra and wavelengths outside the $\mathrm{Ca}$ core without significant residuals of the line blends in the $\mathrm{Ca}$ line wing (see Fig. 5 below). The procedure corrects for the contamination with scattered light from the solar disk inside the local FOV in the direction perpendicular to the limb, but does not account for a possible contamination with stray light from the disk center (see, e.g., Zwaan 1965; Mattig 1971; Martinez Pillet et al. 1990). Using an average disk center profile for the stray-light correction worked worse for our data, partly because the wavelength locations of the spectral lines in the wing of $\mathrm{Ca}$ II $\mathrm{H}$ in the disk center profile did not match to the locations in the stray light beyond the limb. The stray-light contamination close to the limb therefore seems to come mainly from inside the local FOV, as also found by Mattig (1983). The light level in (pseudo)continuum windows of the spectra beyond the limb also was only about $2 \%$ of $I_{\mathrm{c}}$ before the correction.

Intensity normalization. After the correction for the stray-light contamination, the spectra were normalized to absolute units by two coefficients. The first coefficient was derived from the series of disk center measurements to obtain the detector count

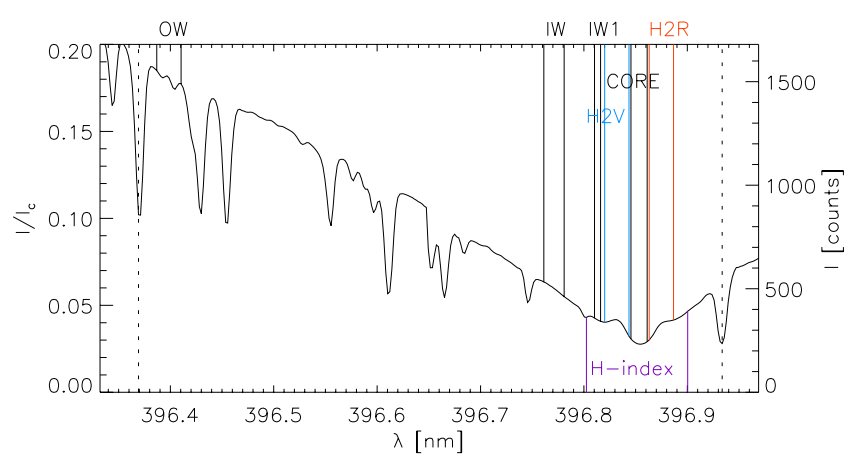

Fig. 2. Average Ca spectrum of the observed FOV (on disk). The locations of specific spectral windows are marked by vertical solid lines. The vertical dashed lines denote two blends whose line-core velocity was additionally measured. The left scale gives the intensity relative to the disk-center continuum, the right scale the detector count value before the normalization.

value in the line wing at disk center at the moment of the observation. After division with this value of about 3000 counts, the spectra are normalized to unity in the line wing on the disk center. To facilitate a conversion to absolute energy units, we then determined the normalization coefficient of an average disk-center spectrum taken on the same day to the FTS atlas (Kurucz et al. 1984) at the same wavelength in the line wing $(I(F T S, 396.4 \mathrm{~nm}) \sim 0.357)$. Because we also have a Ca spectrum on disk center in absolute energy units from an NLTE calculation available (Rezaei et al. 2008), we were then able to convert all relative intensity values to absolute energy units. Figure 2 shows the average spectrum of the observed FOV after all corrections and the normalization; the intensity scale relative to the continuum intensity is given at the left, the actual observed detector count values are provided by the scale at the right. Even with the 13-s integration, the low light level in the Ca core led to only a few hundred counts.

We marked several wavelength windows in the spectrum that will be used below, with increasing wavelength outer wing (OW), inner wing (IW) and inner wing 1 (IW1), $\mathrm{H}_{2 \mathrm{~V}}(396.832 \pm$ $0.012 \mathrm{~nm}$ ), line core (CORE, $396.853 \pm 0.008 \mathrm{~nm}$ ), and $\mathrm{H}_{2 \mathrm{R}}$ $(396.875 \pm 0.012 \mathrm{~nm}$ ) (cf. also the definitions in Cram \& Dame 1983; Lites et al. 1993). The commonly used H-index (an $1 \AA$ wide band around the line core) is marked as well. We did not calculate the integral over the spectral window for all these quantities but the average value relative to $I_{\mathrm{c}}$ instead, which allows an easier comparison with individual spectra or the intensity at some fixed wavelength.

\section{Data analysis and results}

The $\mathrm{Ca}$ II $\mathrm{H}$ line spectra are usually complex-shaped in the interesting wavelength range near the $\mathrm{Ca}$ line core, and no direct analysis tools exist for a derivation of solar atmospheric properties from the spectra, mainly because of the NLTE conditions under which the line core forms. The Ca spectra near and off the limb are even more difficult to analyze than Ca spectra on the disk. This is caused by the effect of the integration along the LOS. On the disk, one can assume that a LOS traverses a very limited number of different structures because of the steep increase in density and thus opacity along the LOS, whereas a LOS near the limb can pierce many more structures in an atmosphere of nearly constant density along the LOS. It turned out, however, that most analysis methods employed on disk spectra 


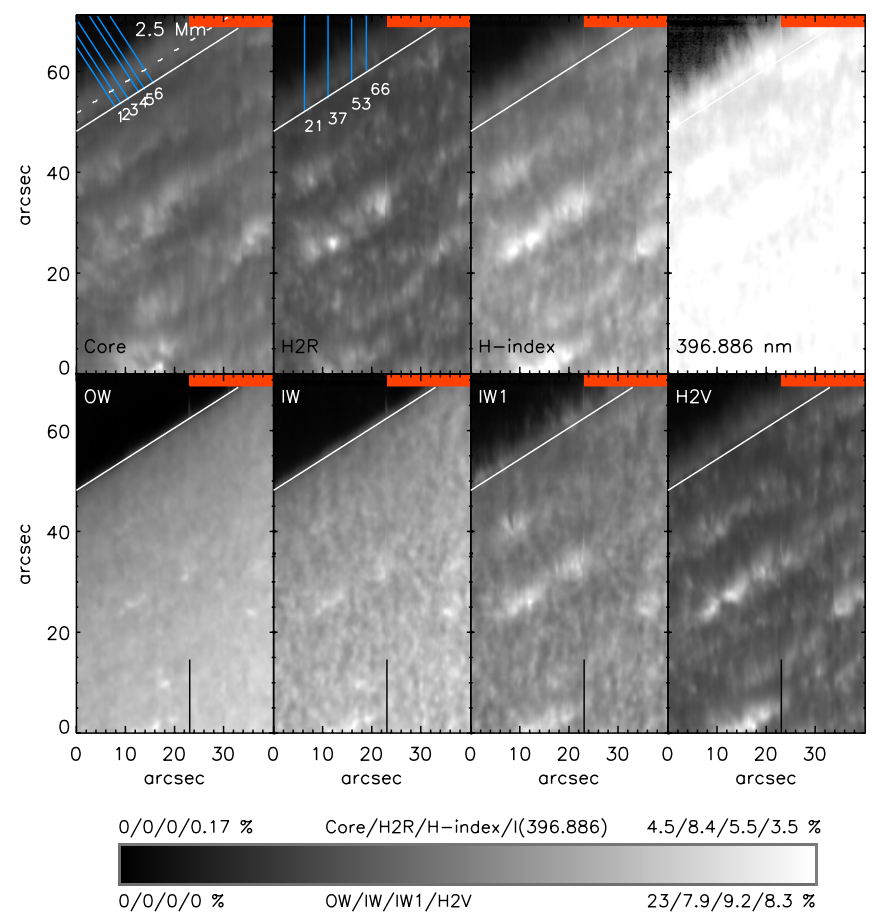

Fig. 3. Overview of the FOV in different spectral windows, denoted at the upper/lower left inside each image. The inclined white line denotes the limb, the black vertical line at the bottom at $x \sim 23^{\prime \prime}$ a jump of the AO. For this reason, the red shaded area in the upper right corner of each image could not be used after alignment to the rest of the FOV. The blue lines in the first two columns of the top row denote cuts through the FOV used later on; the dashed white line in the line-core map marks a limb distance of $2.5 \mathrm{Mm}$. The fourth column in the top row is taken at a single fixed wavelength and thresholded to enhance the visibility of off-limb structures.

(cf. Rezaei et al. 2007, 2008; Beck et al. 2008, 2009) can be used on the limb spectra as well.

\subsection{Full FOV}

FOV in different wavelength ranges. Figure 3 shows how the FOV appears in the various wavelength ranges marked in Fig. 2. We defined the location of the limb in "pseudo-white-light" as the place where the intensity drops below 5\% in the OW map. The (isolated) bright faculae in the OW map are all related to a significant polarization signal in the $630 \mathrm{~nm}$ channel (not shown here). From OW to IW, the on-disk features increase in contrast, and generally have the trend to increase in size as well. The intensity seen beyond the limb is increasing with decreasing distance to the very line core. The map of IW1 is the first one where the structuring changes from roundish shapes to elongated features. Several streaks with high intensity can be seen above the limb that were absent before. The orientation of the streaks is closer to being parallel to the slit than to being perpendicular to the limb in many cases. This could either reflect the true orientation of the underlying solar structures or be somehow caused by the way of data acquisition with a slit-spectrograph system, but the spatial resolution achieved does not allow one a definite conclusion (compare to, e.g., Pasachoff et al. 2009, Fig. 13, or Shoji et al. 2010, Fig. 2). The fine-structure above the limb can also be clearly seen in the intensity map taken at a fixed wavelength (fourth column in the top row) whose display range was thresholded to emphasize the off-limb intensities. In the latter, the offlimb fine-structure actually is more prominent than in the maps of "pure" chromospheric quantities like $\mathrm{H}_{2 \mathrm{~V}}$, line core, and $\mathrm{H}_{2 \mathrm{R}}$. The emission extends furthest above the limb in the line-core map, as is expected, but in general is more uniform there than for $\mathrm{H}_{2 V}$ and $\mathrm{H}_{2 \mathrm{R}}$ (similar to the Fig. 7 of Rutten 2007). If one compares the $\mathrm{H}_{2 \mathrm{~V}}$ and $\mathrm{H}_{2 \mathrm{R}}$ maps with the wavelength-integrated $\mathrm{H}$-index (third column in top row), one finds several occasions where the bright emission in the H-index actually only comes from one of the emission peaks, e.g., at the lower middle near $(x, y)=\left(18^{\prime \prime}, 2^{\prime \prime}\right)$ or near $(x, y)=\left(11^{\prime \prime}, 23^{\prime \prime}\right)$. The H-index does not allow one to distinguish between the two cases.

LOS velocity proxies. We determined the LOS velocities for two of the photospheric blends (dotted lines in Fig. 2) that correspond to the lowest ( $v_{1}, \mathrm{Cr}$ I at $\left.396.369 \mathrm{~nm}\right)$ and highest ( $v_{2}, \mathrm{Fe}_{\mathrm{I}}$ at $396.93 \mathrm{~nm}$ ) forming lines inside the observed spectral range (Beck et al. 2009). For the Ca core, we also determined the location of the minimum intensity in each profile. This method, however, fails from some distance above the limb outward because the $\mathrm{Ca}$ core shows a single emission peak there. For that region, the ratio between $\mathrm{H}_{2 \mathrm{~V}}$ and $\mathrm{H}_{2 \mathrm{R}}$ emission peaks has to be used as proxy for the velocity; Rezaei et al. (2007) discussed the tight relation between the location of the absorption core and the $\mathrm{H}_{2 \mathrm{~V}} / \mathrm{H}_{2 \mathrm{R}}$ ratio (their Fig. 6). The $\mathrm{H}_{2 \mathrm{~V}} / \mathrm{H}_{2 \mathrm{R}}$ ratio still indicates a Doppler shift when the profile reverts to a pure emission pattern, but with the opposite velocity sign. We also calculated the center-of-gravity (COG) of the intensity in an $1.5 \AA$ range around the $\mathrm{Ca}$ line core, but this method is only reliable above the limb where the Ca profiles show a single emission peak. The zero point of the velocity was defined by the average value in the lower half of the FOV. Figure 4 shows the resulting 2D maps for the velocity proxies. The patterns of photospheric and chromospheric velocities are fairly unrelated; the chromospheric velocity pattern also shows no relation to the photospheric OW map (see also Beck et al. 2009). The two maps of the line-core position $\left(v_{\mathrm{Ca}}\right.$, fourth column) and the ratio of $\mathrm{H}_{2 \mathrm{~V}} / \mathrm{H}_{2 \mathrm{R}}$ (fifth column) match in the spatial patterns on the disk, e.g., inside and close to the red contours, even if the amplitude of changes is higher for $v_{\mathrm{Ca}}$. This supports the use of the ratio of $\mathrm{H}_{2 \mathrm{~V}}$ and $\mathrm{H}_{2 \mathrm{R}}$ for the spectra above the limb without any clear absorption core.

Profile shape analysis. For the analysis of the profile shape, we used the same method as in Rezaei et al. (2008), the determination of the number of intensity reversals. The reversals are defined as local maxima of the intensity spectrum in a wavelength range around the line core. The resulting map is displayed in the sixth column of Fig. 4.

In comparison to observations near disk center, the area fraction of double-peaked profiles is slightly enhanced near the limb $(>50 \%)$. This could be an effect of the strongly inclined LOS: upward propagating waves have a smaller LOS component at the location of the FOV, therefore the central absorption core is located at the rest wavelength between the two emission peaks instead of suppressing $\mathrm{H}_{2 \mathrm{R}}$ as happens on disk center. Several coherent larger-scale areas can be found where profiles without reversals exist (black shading, cf. Rezaei et al. 2008), located between the "magnetic" regions as indicated by the increased emission and the polarization signal. From the very limb to about $5 \mathrm{Mm}$ height, the profiles all have at least two reversals (light gray shading). At about $5 \mathrm{Mm}$ there is a rather abrupt transition to single-lobed profiles (dark-gray shading); the height of the transition shows little variation with the spatial position along the limb. We visually inspected the profiles with more than two 


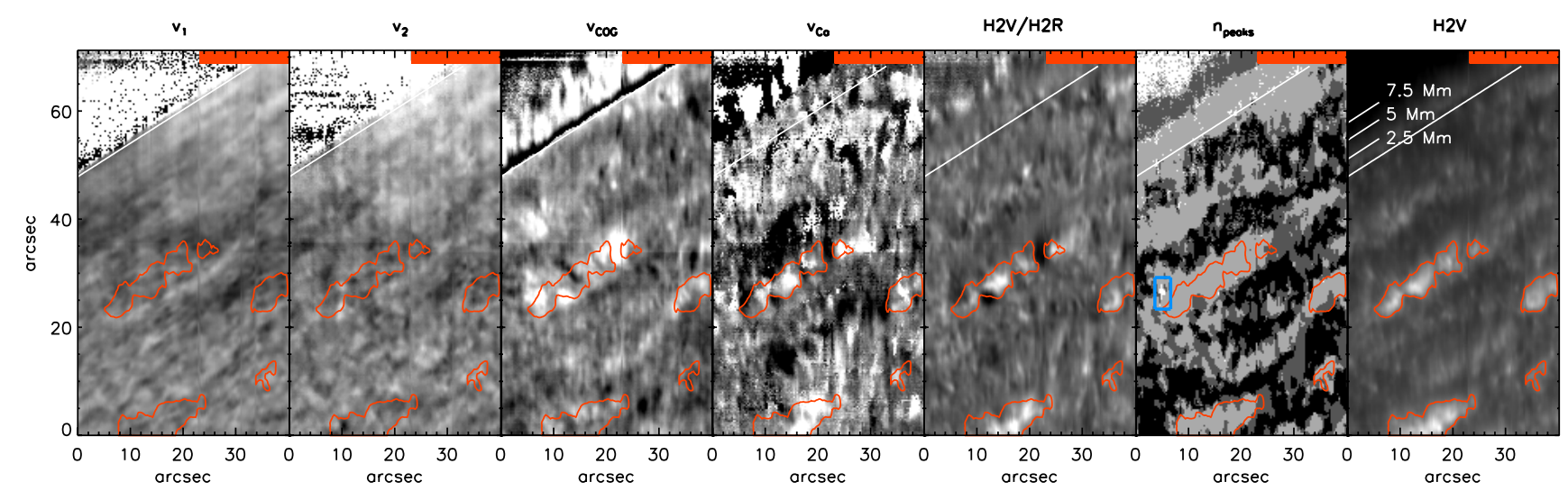

Fig. 4. Left to right: line-core velocity of $396.369 \mathrm{~nm}$, same for $396.93 \mathrm{~nm}, \mathrm{COG}$ of Ca core region, velocity of Ca core, ratio $\mathrm{H}_{2 \mathrm{~V}} / \mathrm{H}_{2 \mathrm{R}}$, number of reversals from 0 (black), 1 (dark gray), 2 (light gray) to 3 or more (white), $\mathrm{H}_{2 \mathrm{~V}}$ intensity. White/black in all velocity maps corresponds to motions away from/toward the observer. The red contour lines denote locations with increased emission. The blue rectangle in the peak map outlines a region of multi-lobed profiles. The short bars parallel to the limb in the $\mathrm{H}_{2 \mathrm{~V}}$ map denote some heights above the limb.

reversals (white). Near and above the limb they turned out to be artifacts created by either the spectral line of Fe I at $396.93 \mathrm{~nm}$ or the noise level, but in one region still on the disk (blue rectangle in the sixth column of Fig. 4 at $\left.(x, y)=\left(5^{\prime \prime}, 25^{\prime \prime}\right)\right)$ they are real. Figure B.1 shows one such profile with several reversals near the line core. It would be interesting to see if numerical simulations like those described by Wedemeyer et al. (2004) or MartínezSykora et al. (2009) yield such profiles in a spectral synthesis (for an initial attempt, see Haberreiter et al. 2010).

\subsection{Average off-limb spectra}

Figure 5 shows the evolution of the spectral shape of $\mathrm{Ca}$ II $\mathrm{H}$ with increasing distance to the limb, $d$. The spectra were averaged over height bins of $362 \mathrm{~km}(\equiv 0$.'5) and across the FOV; except for the two profiles closest to the limb, only every second profile is plotted in the uppermost panel. The profiles are labeled with the corresponding height. Close to the limb, the average spectra are double-peaked with only a small asymmetry between $\mathrm{H}_{2} \mathrm{~V}$ and $\mathrm{H}_{2 \mathrm{R}}$. The line-core intensity slightly increases when going outward, whereas the emission peaks decrease. At a height of about 4-5 Mm above the limb this leads to profiles without a central absorption, showing a broad plateau instead. The central emission decreases slower than that near the wavelengths of $\mathrm{H}_{2 \mathrm{~V}}$ and $\mathrm{H}_{2 \mathrm{R}}$ and a single-peaked broad Gaussian remains above about $5 \mathrm{Mm}$. Extrapolating the intensities measured by Dunn et al. (1968) during an eclipse, Linsky \& Avrett (1970) suggested that at a height of about 5-6 Mm above the limb the ratio of the $\mathrm{Ca}$ II $\mathrm{H}$ and $\mathrm{K}$ lines should approach a value of 2 , implying that the lines become optically thin. Then the central absorption disappears, leaving a single emission core, which agrees with our observed spectra. The profiles compare well to those given by Zirker (1962, ZI62, his Fig. 3), generally having a slightly lower intensity. In a recent publication, Judge \& Carlsson (2010) provided synthetic $\mathrm{Ca}$ spectra for a simplified model of spicules near and off the limb. The spectra shown in their Fig. 3 seem to match the general properties found in our observations well, showing a similar transition from double-peaked emission to a single-peak emission of decreasing width with height.

The Fe II line near $396.94 \mathrm{~nm}$ reverts to emission, as do some of the other blends, but none as strong as this one (see Engvold \& Halvorsen 1973; Rutten \& Stencel 1980). This line is consequently interesting for detailed quantitative analyses as well (Lites 1974; Watanabe \& Steenbock 1986), to avoid solely using the Ca II H line core, even if Cram et al. (1980) found a photospheric origin for the emission in this line.

All wavelengths that show emission have a (more or less) pronounced maximum of intensity whose height above the limb increases the closer the wavelength is to the line core (middle panel). This is similar to the findings of White (1963) for the He I $\mathrm{D}_{3}$ line or for the He $\mathrm{I}$ line at $1083 \mathrm{~nm}$ (Schmidt et al. 1994; Penn \& Jones 1996; Sánchez-Andrade Nuño et al. 2007). The maximum intensity occurs at layers higher than found using intensity contribution functions for these wavelengths (e.g., Rezaei et al. 2008; Beck et al. 2009). This is caused by the stretched optical depth scale in a LOS nearly parallel to the solar surface.

We determined the energy contained in the radiation emitted by the line-core region by integrating the spectra over the wavelength range from $396.72 \mathrm{~nm}$ to $396.96 \mathrm{~nm}$ (bottom panel). The total energy radiated by the $\mathrm{Ca}$ II $\mathrm{H}$ line smoothly decreases from about $400 \mathrm{Wm}^{-2}$ ster $^{-1}$ near the limb on the disk to about $100 \mathrm{Wm}^{-2}$ ster $^{-1}$ at a height of $4 \mathrm{Mm}$ (cf. Judge \& Carlsson 2010). The values provided by Beckers (1968, BE68) for heights of 4 and $6 \mathrm{Mm}$ in his Table 11 match our values when divided by a factor of 5.5 (red vertical bars). This is presumably because of his scaling of the total intensity to an ad-hoc assumed spicule size, whereas we use spectra of single pixels corresponding to a $0 . .5 \times 0 ., 3$ area.

Figure 6 shows the full set of average off-limb spectra. In the top panel we normalized each row of the spectrum separately to its maximum intensity between $396.763 \mathrm{~nm}$ and $396.920 \mathrm{~nm}$ to highlight the shape. We then used three different methods to estimate the line width of the spectra. For spectra up to about $4 \mathrm{Mm}$, two distinct emission peaks are seen whose position can be determined (blue dots). After the emission peaks have merged to a single broad peak, we determined the location where the intensity drops to $50 \%$ of the maximum intensity near the line core and fitted a Gaussian to the core region of the spectra as well to derive the full width at half maximum ( $F W H M$, black dots). The latter two methods gave similar results, but cannot be employed below a limb distance of about $2 \mathrm{Mm}$ because the increasing intensity of the line wings makes it impossible to define where the contribution of the line core ends. For heights below $2 \mathrm{Mm}$ one could assume that the $F W H M$ will follow the behavior of the emission peaks, which would yield the black dashdotted line in the lower panel of Fig. 6. The FWHM of the emission signal near the line core, including the $\mathrm{H}_{2 \mathrm{~V}}$ and $\mathrm{H}_{2 \mathrm{R}}$ emission peaks, increases slightly from about $90 \mathrm{pm}$ at the limb to about $97 \mathrm{pm}$ at $2 \mathrm{Mm}$ height, then decreases by a factor of 2 

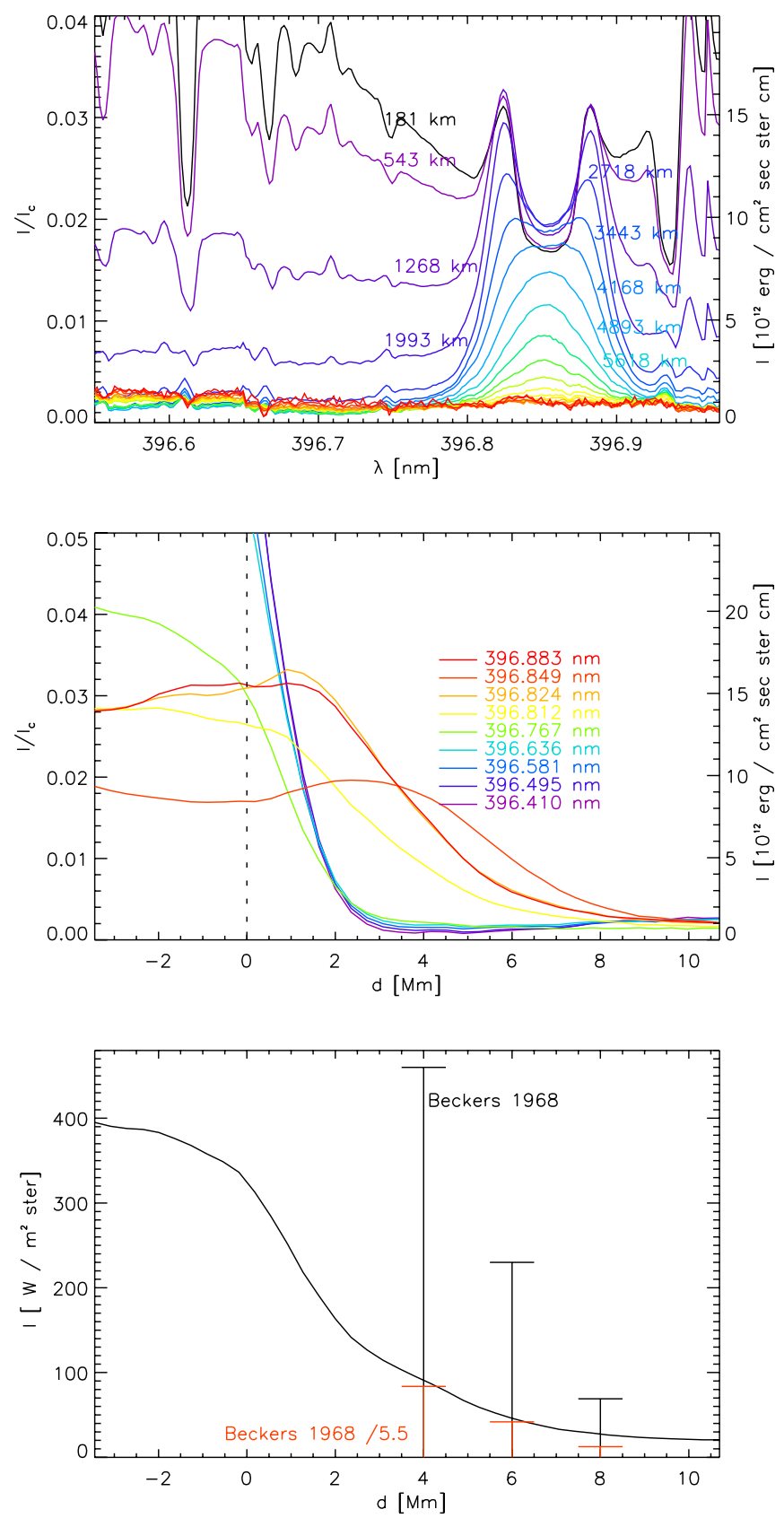

Fig. 5. Top panel: average off-limb spectra of $\mathrm{Ca}$ II $\mathrm{H}$ with increasing limb distance $d$. Middle panel: intensity variation with limb distance for the wavelengths marked in Fig. 6. The limb is denoted by a vertical black dashed line. The right scale uses the same units as in Zirker (1962). Bottom: the energy of the radiation integrated over the line-core region. The black vertical bars denote the energy as given by BE68, and after division by a factor of 5.5 , respectively (red).

until about 5 Mm (cf. for instance Makita 2003, his Fig. 9). The subsequent increase of the width for layers above $5 \mathrm{Mm}$ is presumably not real because the intensity reaches the noise level. Our values agree to first order with the line widths provided in ZI62(83 pm@5.1 Mm and 68 pm@6.4 Mm) or in BE68. The latter defined the width $w$ as $F W H M / \lambda$ (see the scale at right) and obtained $w=1.4$ at $5 \mathrm{Mm}$ (his Table 11).

The observations beyond the limb offer the possibility to define the FWHM of the $\mathrm{Ca}$ II $\mathrm{H}$ line for a Sun as a star spectrum. The values near a height of $2 \mathrm{Mm}$ in Fig. 6 suggest that the FWHM is larger than the peak separation by a factor of about 1.7 (see also Sivaraman et al. 1987, their Figs. 6 and 8
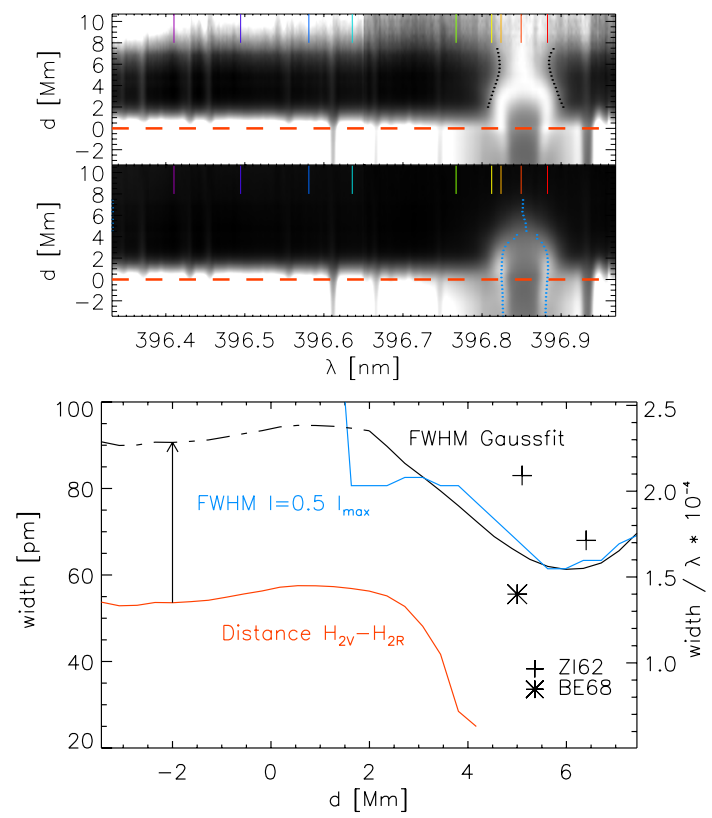

Fig. 6. Top: the full average off-limb spectrum normalized row by row; the wavelengths used in the middle panel of Fig. 5 are marked by short vertical lines at the top. Middle panel: the same spectrum without additional normalization. The blue dots indicate the locations of the emission peaks, the black dots the $F W H M$. The red horizontal dashed lines denote the location of the limb. Bottom panel: FWHM from a fit of a Gaussian (black), from the location of $50 \%$ of the maximum intensity (blue), and the distance between the emission peaks (red). Crosses and asterisks mark the values given by ZI62 and BE68, respectively.

yield a factor of about 1.4). The distance between the $\mathrm{H}_{2 \mathrm{~V}}$ emission peak and the line core in the FTS atlas spectrum is about $15.6 \mathrm{pm}$. Because the position of $\mathrm{H}_{2 \mathrm{R}}$ cannot be reliably determined in the profile, we assume that the peak separation should be twice as large. This finally gives a $F W H M$ at disk center of $53 \mathrm{pm}$, corresponding to $40 \mathrm{~km} \mathrm{~s}^{-1}$ (logarithmic value of 1.6). Wilson \& Vainu Bappu (1957) give a range of 33-39 $\mathrm{km} \mathrm{s}^{-1}$ for $\mathrm{Ca}$ spectra in plage where the emission core is more pronounced and can be separated from the wing contribution (see also Teplitskaja \& Efendieva 1973; Jebsen \& Mitchell 1978; Oranje 1983; Sivaraman et al. 1996). The prediction of Wilson \& Vainu Bappu (1957) for the Sun as a star line width of Ca II H or $\mathrm{K}$ is 1.56 , derived from their curve of what later was termed the Wilson-Bappu effect (cf. also Stencel 2009).

\subsection{Individual off-limb spectra}

We discuss the spatially resolved spectra on cuts parallel to the limb, along the slit, and perpendicular to the limb.

Spectra parallel to the limb - horizontal variation. Figure 7 shows the observed spectra on cuts parallel to the limb (some examples of the corresponding heights above the limb are marked in the right most panel of Fig. 4). The maximal intensity at each height is denoted in the lower right corner. One outstanding pattern throughout all heights are the Doppler excursions of the absorption core. For many spatial locations, the blue and red shifts can be traced through all heights. They are especially visible in the height range from 2.5 to $4 \mathrm{Mm}$, yielding a clear zig-zag pattern. At lower layers, they can be seen in the intensity difference between the $\mathrm{H}_{2 \mathrm{~V}}$ and $\mathrm{H}_{2 \mathrm{R}}$ emission peaks which brighten and darken with the shifts of the absorption core $(d=1$ to $2 \mathrm{Mm}$, 


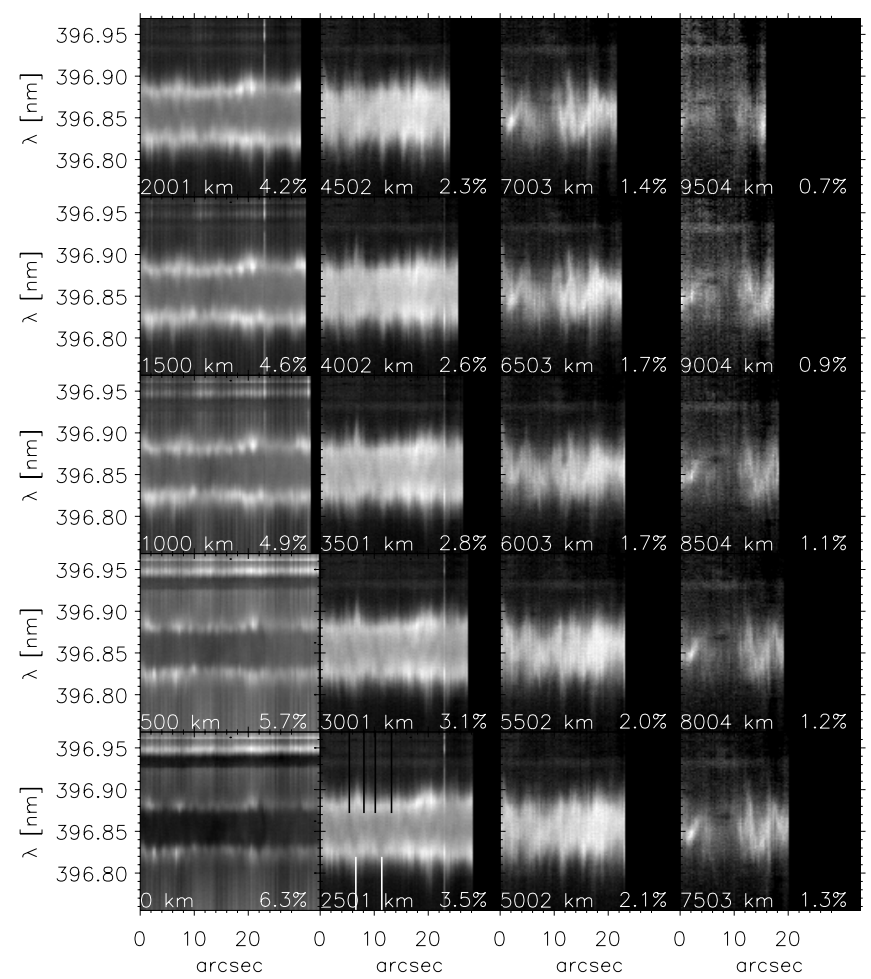

Fig. 7. Spectra on cuts parallel to the limb. The height of the cut is denoted in the lower left, the maximum intensity at lower right; the $x$-axis gives the position along the limb, the $y$-axis the wavelength. For the spectra at $2.5 \mathrm{Mm}$, the locations of Doppler excursions of the absorption core to the blue/red are denoted by white/black vertical bars.

$\left.x \sim 20^{\prime \prime}\right)$. This phenomenon has often been called line tilt (e.g., Beckers 1968; Pasachoff et al. 1968). An extensive discussion of possible explanations of these line tilts is given by Rompolt (1975).

Six examples of prominent Doppler excursions are marked in the spectra of the cut parallel to the limb at $2.5 \mathrm{Mm}$ height (second column, bottom row). The resulting line tilt corresponds to a shift of the central absorption core that partially suppresses one of the emission peaks while enhancing the other one. The variation of the strength of the emission peaks is even more pronounced at about $x=20^{\prime \prime}$. The evolution of the spectra from a red shift to a blue shift and back takes part on a typical scale of $4-6$ profiles ( $\equiv 1.2$ to $1.8^{\prime \prime}$ ). Figure B.2 shows the individual spectra around the positions marked in Fig. 7. Figure 8 displays the appearance of the line-core shifts in the velocity proxies and intensity bands. They are clearly mirrored in the intensity difference of the $\mathrm{H}_{2 \mathrm{~V}}$ and $\mathrm{H}_{2 \mathrm{R}}$ emission peaks and the line-core velocity (top panel). The velocity amplitude is about $\pm 4 \mathrm{~km} \mathrm{~s}^{-1}$. There is a trend for local intensity maxima of the H-index, which is insensitive to the Doppler shifts, to occur between the maximal and minimal velocities (dashed lines in the bottom panel).

Spectra perpendicular to the limb - vertical variation. The spectra shown in the following Figs. 9 and B.3 were taken along the cuts marked in the top left panels of Fig. 3. The six rays perpendicular to the limb, labeled 1 to 6 , were chosen to intersect the line of $2.5 \mathrm{Mm}$ distance to the limb such that they correspond to the locations of the large Doppler excursions marked in Fig. 7. The spectra along these cuts are shown in Fig. 9. The Doppler excursions are seen on the one hand as bright "branches" to the blue and red; on the other hand, they also appear in the variation

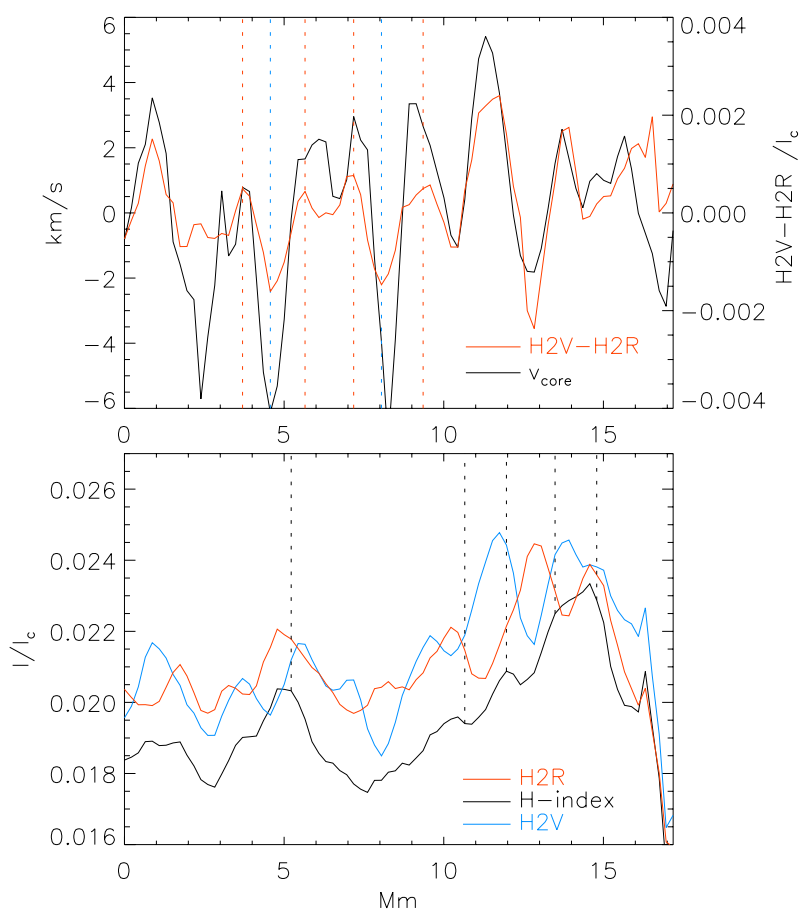

Fig. 8. Appearance of the Doppler excursions at $2.5 \mathrm{Mm}$ in intensity and velocity. Top: velocity proxies. Red: difference of $\mathrm{H}_{2 \mathrm{~V}}$ and $\mathrm{H}_{2 \mathrm{R}}$. Black: position of the line core. The blue and red dashed vertical lines denote the locations of blue/red shifts. Bottom: intensity of $\mathrm{H}_{2 \mathrm{~V}}$ (blue), $\mathrm{H}_{2 \mathrm{R}}$ (red), and the $\mathrm{H}$-index (black). The vertical black dashed lines indicate local maxima of the H-index. The abscissa is in $\mathrm{Mm}$.

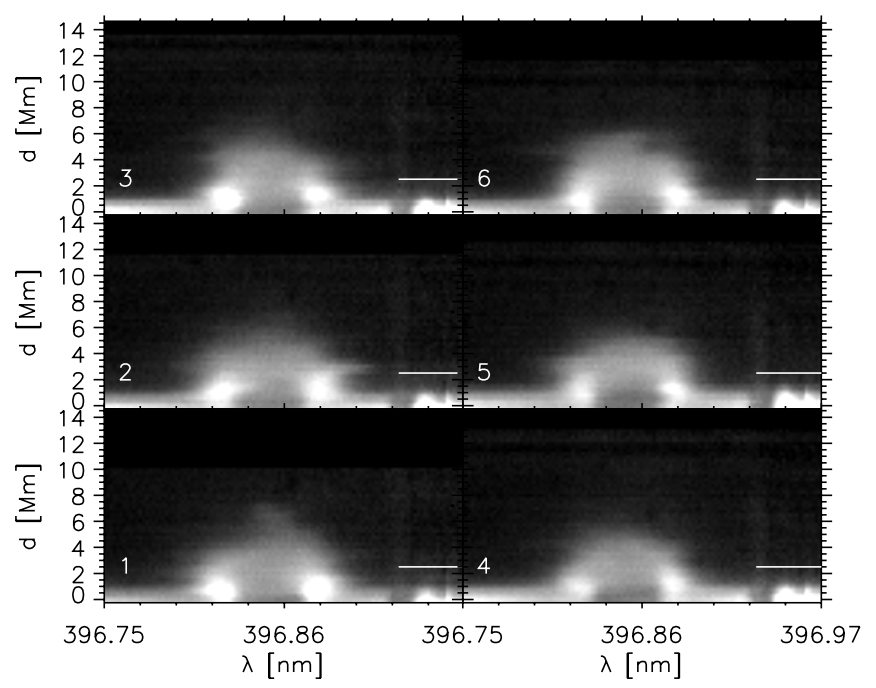

Fig. 9. Spectra corresponding to the cuts 1 to 6 marked in Fig. 3. The limb is at bottom, the $y$-axis gives the distance $d$ to the limb. The horizontal white bars at the right indicate a height of $2.5 \mathrm{Mm}$. The display is thresholded at $3 \%$ of $I_{\mathrm{c}}$ for better visibility.

of the relative intensities of $\mathrm{H}_{2 V}$ and $\mathrm{H}_{2 \mathrm{R}}$. In some cases, the Doppler shifts show an oscillatory pattern not only in the horizontal direction (as seen by the variation between ray 1,2 , and 3 ), but in the vertical direction as well, e.g., for the case of ray 2 , giving the spectra a faint similarity with a Christmas tree. The individual spectra of cut no. 2 are shown in Fig. B.4.

The other cuts were taken along the slit for two reasons. First, the spectra of a slit position are taken simultaneously, so only a temporal evolution faster than the 13-s integration time can play a role. Second, many of the elongated structures seen in for instance the IW1 map of Fig. 3 are rather oriented along the slit 


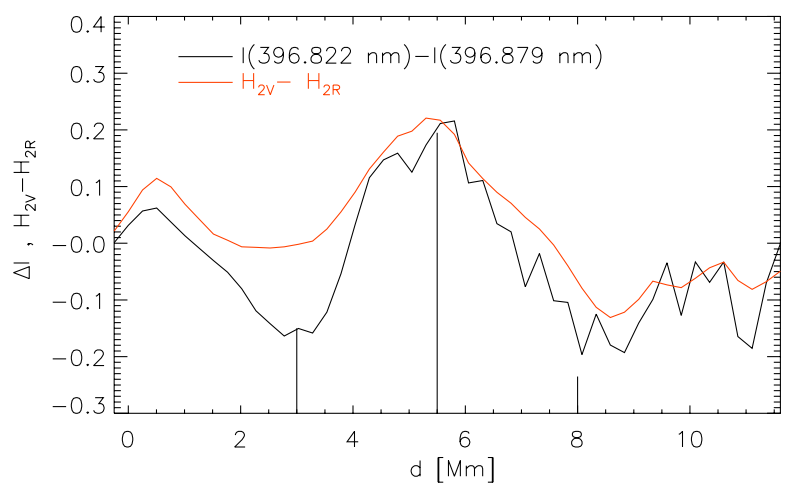

Fig. 10. Vertical variation of intensities along cut no. 2. Black: difference of intensities at $396.822 \mathrm{~nm}$ and $396.879 \mathrm{~nm}$. Red: difference of $\mathrm{H}_{2 \mathrm{~V}}$ and $\mathrm{H}_{2 \mathrm{R}}$.

than perpendicular to the limb. For the cuts along the slit, we always marked one of the scan steps with its number in Fig. 3, to be able to relate the shape of the spectra on that location to a specific prominent feature in the $2 \mathrm{D}$ maps of the FOV. Because the general patterns in these spectra are similar to those of Fig. 9, we moved them to the appendix. Repeated Doppler excursions to the red and the blue along the vertical direction can be seen in several examples; the largest Doppler shifts are usually seen at some distance (about 2-6 Mm) above the limb. The Doppler excursions are coherent over some scan positions, e.g., for the scan steps 65 to 68 at $y=13^{\prime \prime}$ (Fig. B.3), which also implies a lower limit for the duration of about $50 \mathrm{~s}$ in addition to the spatial extent of $1-2^{\prime \prime}$. Some features like the strong brightening of $\mathrm{H}_{2 \mathrm{R}}$ in scan step 69 at $y=11^{\prime \prime}$ (also in Fig. B.3) fade away (toward subsequent steps) or significantly change shape (toward previous scan steps) over 2-3 steps (三0.'6-0!'9) only, with 0.'6 being the technical lowermost possible limit for the resolution. The individual spectra of the scan step 66 are shown in Fig. B.5.

The spatial extent of the Doppler variations along the local vertical is shown in Fig. 10 using the profiles of cut no. 2 (cf. also the map of $\mathrm{H}_{2 \mathrm{~V}} / \mathrm{H}_{2 \mathrm{R}}$ in Fig. 4 at $\left.(x, y)=\left(6^{\prime \prime}, 50^{\prime \prime}\right)\right)$. We calculated the intensity difference of $\mathrm{H}_{2 \mathrm{~V}}$ and $\mathrm{H}_{2 \mathrm{R}}$ in each profile (red line), and also created a single-wavelength "Dopplergram" by subtracting $I(\lambda=396.879 \mathrm{~nm})$ from $I(\lambda=396.822 \mathrm{~nm})$ for comparison (black line). The latter two wavelengths sample only the faint "branches" to the blue and red and are therefore partly insensitive to the location of the central absorption core measuring shifts of the complete emission pattern, but basically yield the same spatial variation as the emission peaks. Owing to the low intensity of the "branches", the other velocity proxies do not capture the Doppler shifts well for these profiles. To improve the visibility of the Doppler shifts, we removed the intensity decrease with distance from the limb by dividing the differences with the sum of the two terms. The recursive Doppler oscillations then occur with a separation of about 2.5-3 Mm (black vertical lines) along the local vertical. It cannot be excluded, however, that the LOS crossed more than one distinct structure and sampled its contribution to the spectra. The repetitive Doppler excursions perpendicular to the limb are in general less pronounced than the variation on cuts parallel to the limb.

\subsection{Statistics of Doppler shifts}

Because the $\mathrm{Ca}$ line profile looses its central absorption core at a height of $5 \mathrm{Mm}$ above the limb and other LOS velocity proxies like the difference of $\mathrm{H}_{2 \mathrm{~V}}$ and $\mathrm{H}_{2 \mathrm{R}}$ do not yield a quantitative velocity measure, we restricted the analysis of the statistics of Doppler shifts to a maximum height of $5 \mathrm{Mm}$. The unsigned velocity ranges up to about $15 \mathrm{~km} \mathrm{~s}^{-1}$, with a mean value of $3.2 \mathrm{~km} \mathrm{~s}^{-1}$. The root-mean-square (rms) for the signed velocity is about $4 \mathrm{~km} \mathrm{~s}^{-1}$, which agrees with the $3.8 \mathrm{~km} \mathrm{~s}^{-1}$ given by BE68 for a height of $6 \mathrm{Mm}$. We find that the rms velocity of the photospheric blends and the Ca core increases toward the limb, similar to the behavior of the line width shown in Fig. 6.

\section{Summary}

We investigated a set of spectra of $\mathrm{Ca}$ II $\mathrm{H}$ inside a FOV that extended some $20^{\prime \prime}$ beyond the solar limb. We estimate that we achieved a spatial resolution of about $1-1.5^{\prime \prime}$ with permanent image stabilization to better than about 0.5 . The data set allowed us to retrieve average off-limb Ca spectra with a well-defined limb distance. The average spectra show a smoothly increasing width of the Ca line core up to about 2-3 Mm height above the limb. At a height of $5 \mathrm{Mm}$, the line width starts to reduce strongly. The emission peaks are generally symmetric. Above a limb distance of $5 \mathrm{Mm}$, the profile shape is similar to a single Gaussian. Wavelengths in and near the core show a local intensity maximum above the limb, whose height decreases with separation from the very core, as expected from intensity contribution functions. The found variation with limb distance of the intensity, the integrated emission, and the line width should be reproduced by theoretical models, providing boundary conditions for, e.g., the microturbulent velocity and the temperature stratification of the chromosphere.

Individual profiles from beyond the limb show ubiquitously large Doppler shifts, visible either as a clear shift of the absorption core or as a pronounced asymmetry of $\mathrm{H}_{2 \mathrm{~V}}$ and $\mathrm{H}_{2 \mathrm{R}}$. On cuts parallel to the limb, the sign of the velocity reverses on the smallest spatial scales resolved in the data $\left(\sim 1.5^{\prime \prime}\right)$. The velocity amplitude as determined from the position of the absorption core is about $\pm 4 \mathrm{~km} \mathrm{~s}^{-1}$. Cuts perpendicular to the limb or along the slit show in some cases a similar pattern of sign reversal of the velocity. The changes of the velocity sign often extend over a few arcseconds in the direction perpendicular to the limb and flank slight enhancements of the total emission as given by the wavelength-integrated $\mathrm{H}$-index.

\section{Discussion}

We have obtained medium-resolution slit spectra of $\mathrm{Ca}$ II $\mathrm{H}$ near and above the limb. The series of disk center observations with identical exposure times obtained during the observing day allowed us to obtain an absolute intensity calibration whose accuracy is only limited by the applied stray-light corrections. The coefficient $\alpha$ of the stray-light correction directly scales the total intensity by the subtraction of the average profile. This affects the spectra on the disk most strongly because beyond the limb the stray-light coefficient is rapidly decreasing (Fig. 1). Beyond the limb, the intrinsic emission in the line core with an intensity of about $1 \%$ at a height of $5 \mathrm{Mm}$ (cf. Fig. 5) dominates over at least the residual stray light after the correction. At heights above about $9 \mathrm{Mm}$, the intensity in the line wing and the line core equalize. The remaining intensity in the core of the line (and also all other wavelengths) is presumably only caused by the residual stray-light level because the chromosphere itself has a finite extent of a few Mm. We tested different values of $\alpha$ and of the off-limb correction, but the resulting intensity in the off-disk emission was roughly independent of the exact way of stray-light correction (see Beck et al. 2011). 
Our spectroscopic observations were obtained with real-time image stabilization and correction by an $\mathrm{AO}$ system, with a residual image motion below 1" (see Appendix A). For this, no dedicated device like a limb-tracker as suggested by Pasachoff et al. (2009) is necessary, it is sufficient if adaptive optics and the spatial scanning for a spectrograph or the selection of the FOV to be observed, respectively, are independent of each other ${ }^{2}$. The image stability and the complete coverage of the height range allowed us to sample the spectra continuously with height with a sampling of about 0.'3. In some older observations (e.g., Zirker 1962; Pasachoff et al. 1968, PA68), the spatial sampling was significantly lower or even reduced to two height levels only in the latter case. The Doppler shifts have a clear signature in our offlimb spectra, appearing in two different shapes: displacements of the absorption core relative to the emission peaks, and shifts of the complete profile seen as faint "whiskers" to the red and blue of the line core. In the spectra used by PA68, no displacements of the absorption core were seen (compare their Fig. 11 with Fig. B.2). We relate this to the spatial resolution achieved in our observations because in the average spectra or for a spatial smearing over $2-3^{\prime \prime}$ the oppositely directed Doppler shifts cancel out again.

The observed width of the Ca II H (or K) line can presumably not be caused only by different Doppler shifts of unresolved sub-structures (Athay 1961; Athay \& Bessey 1964). Many multiline observations of limb structures (e.g., Krat \& Krat 1971; Alissandrakis 1973; Athay \& Bessey 1964; Pasachoff et al. 1968; Socas-Navarro \& Elmore 2005) also implied that other processes than thermal Doppler broadening have to be present because the observed line widths of $\mathrm{Ca}$ and other elements could not be reproduced assuming the same temperature and its corresponding thermal broadening. In our case, the line width of the average off-limb profiles shows a smooth variation with an abrupt change at about $5 \mathrm{Mm}$ limb distance. The height of this transition is fairly constant all across the FOV in the spatially resolved spectra. This suggests an optical depth effect caused by absorbing matter in front of an emission background. Eventually the difference between the two type of "wide" (large line width) and "narrow" (small line width) spicules defined by BE68 marks the difference between optically thin and thick structures, where only for the optically thick structures a sufficient self-absorption is present.

The origin of the observed relation (Fig. 8) between enhancements of the wavelength-integrated $\mathrm{H}$-index - which to first order is insensitive to Doppler shifts of the line core - and the oppositely directed LOS velocities that flank them is unclear at first. The pattern is not only seen at the height of $2.5 \mathrm{Mm}$ shown in Fig. 8, but continues over a height range of about $5 \mathrm{Mm}$ (cf. the maps of the $\mathrm{H}$-index in Fig. 3 and that of $\mathrm{H}_{2 \mathrm{R}} / \mathrm{H}_{2 \mathrm{~V}}$ in Fig. 4 at $\left.(x, y)=\left(20^{\prime \prime}, 62^{\prime \prime}\right)\right)$.

There are several possible explanations for the relation between the velocity and intensity pattern. One is that the corresponding solar structures are rotating as a rigid body, which was invoked recently for explaining the observation of a macrospicule by Kamio et al. (2010). Other explanations are waves propagating along the field lines of an intrinsically twisted flux tube (Avery 1970) or waves traveling at an angle to the orientation of the tube axis, the twisting motion of field lines as suggested by the high-resolution, high-cadence $\mathrm{Ca}$ imaging data from HINODE (Suematsu et al. 2008), or helical kink waves (Zaqarashvili \& Skhirtladze 2008). The Doppler shift pattern

\footnotetext{
2 This is a crucial point for any AO-supported observations near the limb that deserves attention in telescope designs.
}

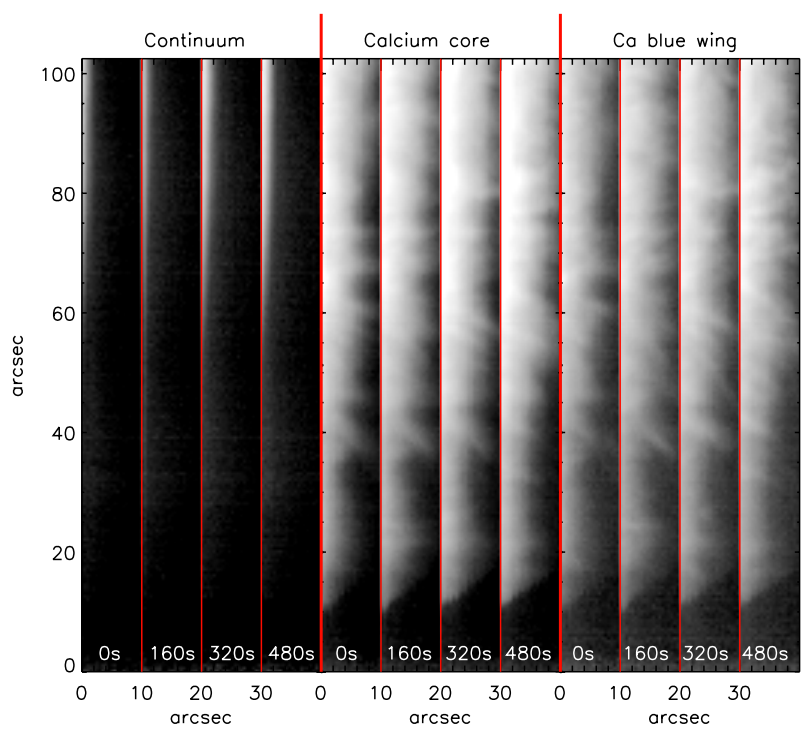

Fig. 11. Example of a time-series of Ca II H spectra taken in July 2010. Left: continuum intensity. Middle: line-core intensity. Right: intensity to the blue of the line core. Exposure time per scan step was $6 \mathrm{~s}$. All images are displayed in logarithmic scale.

caused by a helical wave propagation along a central flux tube would presumably be different from the sausage soliton solution proposed by Zaqarashvili et al. (2010), which would have a maximum velocity amplitude at the center of the brightening instead of the velocity sign reversal on the sides. In view of the limited spatial resolution and the low statistics of our data, we refrain from trying to give a definite answer, however.

Our observations have one drawback for the interpretation of the findings. The orientation of the slit was to first order perpendicular to the limb. Even with the image correction by AO, this increases the stray-light contamination in the off-limb spectra because the on-disk spectra have an intensity higher by an order of magnitude. Observations with the slit parallel to the limb are therefore to be preferred, possibly also a set of repeated scans to allow for a more reliable identification of features. Such data were obtained during an observation campaign in July 2010, but their analysis is still pending. Figure 11 shows an example of the improvement in spatial resolution that is to be expected; the corresponding data have not been corrected for any stray light yet.

The $\mathrm{Ca}$ II $\mathrm{H}$ line is less accessible from the theoretical side than the Ca II IR lines because of the partial redistribution effects inside the spectral line (Heggland et al. 2009; Leenaarts et al. 2009), but it still is easier to handle than $\mathrm{H}_{\alpha}$ (Carlsson \& Stein 2002; Leenaarts et al. 2007). Because many recent results are based on data obtained with the broad-band $\mathrm{Ca}$ filter imaging of HINODE without spectral resolution, it seems worthwhile to cross-check the features in these observations with spectra, eventually from a new solar space mission like Solar-C. A spectral synthesis from simulations like described in Martínez-Sykora et al. (2009) would also be a possibility; or a direct comparison with the synthetic spectra recently presented in Judge \& Carlsson (2010).

Our data set (41 MB) is available online ${ }^{3}$ for free use. This will hopefully raise some more interest in the development of analysis methods for the $\mathrm{Ca}$ II $\mathrm{H}$ line or obtaining high$\mathrm{S} / \mathrm{N}$ slit spectra even in modern times, despite the claim in the

\footnotetext{
http://www3.kis.uni-freiburg.de/ rrezaei/ spiculedata/ or at http://cdsarc.u-strasbg.fr/viz-bin/ qcat?J/A+A/531/A173.
} 
introduction of Leenaarts et al. (2010) that only 2D imaging spectroscopy and spectropolarimetry is "adequate" for studying the chromosphere, or the similar one in the conclusions of Judge et al. (2010).

\section{Conclusions}

Observations near the solar limb with a real-time correction by adaptive optics are possible with medium to high spatial resolution when the AO system allows one to relocate the observed FOV from the AO lock point. Slit-spectrograph observations with their full line-spectra provide the information on both the thermal and dynamical state of the atmosphere, where the information on the Doppler shifts allows different scenarios such as helical or sausage wave modes to be distinguished. The average off-limb spectra can finally be used to test static atmosphere models because above the limb it is ensured that they only sample chromospheric height layers.

Acknowledgements. The VTT is operated by the Kiepenheuer-Institut für Sonnenphysik (KIS) at the Spanish Observatorio del Teide of the Instituto de Astrofísica de Canarias (IAC). The POLIS instrument has been a joint development of the High Altitude Observatory (Boulder, USA) and the KIS. C.B. acknowledges partial support by the Spanish Ministry of Science and Innovation through project AYA2010-18029 (Solar Magnetism and Astrophysical Spectropolarimetry). R.R. acknowledges partial support by the Deutsche Forschungsgemeinschaft under grant SCHM 1168/8-2. We thank B. Lites for discussions on the topic of stray light.

\section{References}

Alissandrakis, C. E. 1973, Sol. Phys., 32, 345

Athay, R. G. 1961, ApJ, 134, 756

Athay, R. G., \& Bessey, R. J. 1964, ApJ, 140, 1174

Avery, L. W. 1970, Sol. Phys., 13, 301

Balasubramaniam, K. S., Christopoulou, E. B., \& Uitenbroek, H. 2004, ApJ, 606, 1233

Beck, C., Schlichenmaier, R., Collados, M., Bellot Rubio, L., \& Kentischer, T. 2005a, A\&A, 443, 1047

Beck, C., Schmidt, W., Kentischer, T., \& Elmore, D. 2005b, A\&A, 437, 1159 Beck, C., Schmidt, W., Rezaei, R., \& Rammacher, W. 2008, A\&A, 479, 213 Beck, C., Khomenko, E., Rezaei, R., \& Collados, M. 2009, A\&A, 507, 453

Beck, C., Rezaei, R., \& Fabbian, D. 2011, A\&A, submitted

Beckers, J. M. 1968, Sol. Phys., 3, 367 (BE68)

Biermann, L. 1948, Zeitsch. Astrophys., 25, 161

Carlsson, M., \& Stein, R. F. 1997, ApJ, 481, 500

Carlsson, M., \& Stein, R. F. 2002, ApJ, 572, 626

Cauzzi, G., Reardon, K. P., Uitenbroek, H., et al. 2008, A\&A, 480, 515

Centeno, R., Trujillo Bueno, J., \& Asensio Ramos, A. 2010, ApJ, 708, 1579

Cram, L. E., \& Dame, L. 1983, ApJ, 272, 355

Cram, L. E., Lites, B. W., \& Rutten, R. J. 1980, ApJ, 241, 374

Dunn, R. B., Evans, J. W., Jefferies, J. T., et al. 1968, ApJS, 15, 275

Engvold, O., \& Halvorsen, H. D. 1973, Sol. Phys., 28, 23

Fontenla, J. M., Avrett, E., Thuillier, G., \& Harder, J. 2006, ApJ, 639, 441

Fontenla, J. M., Balasubramaniam, K. S., \& Harder, J. 2007, in The Physics of

Chromospheric Plasma, ed. P. Heinzel, I. Dorotovic, \& R. J. Rutten, 499

Fossum, A., \& Carlsson, M. 2005, Nature, 435, 919

Haberreiter, M., Finsterle, W., McIntosh, S., \& Wedemeyer-Boehm, S. 2010,

Mem. Soc. Astron. Italiana, 81, 782

Heggland, L., De Pontieu, B., \& Hansteen, V. H. 2009, ApJ, 702, 1

Jebsen, D. E., \& Mitchell, Jr., W. E. 1978, Sol. Phys., 57, 309

Judge, P. G., \& Carlsson, M. 2010, ApJ, 719, 469

Judge, P. G., Tritschler, A., Uitenbroek, H., et al. 2010, ApJ, 710, 1486
Kalkofen, W. 1996, ApJ, 468, L69

Kamio, S., Curdt, W., Teriaca, L., Inhester, B., \& Solanki, S. K. 2010, A\&A, 510, L1

Kosugi, T., Matsuzaki, K., Sakao, T., et al. 2007, Sol. Phys., 243, 3

Krat, V. A., \& Krat, T. V. 1971, Sol. Phys., 17, 355

Kurucz, R. L., Furenlid, I., Brault, J., \& Testerman, L. 1984, Solar flux atlas from 296 to $1300 \mathrm{~nm}$ (NSO, Sunspot, NM)

Leenaarts, J., Carlsson, M., Hansteen, V., \& Rutten, R. J. 2007, A\&A, 473, 625

Leenaarts, J., Carlsson, M., Hansteen, V., \& Rouppe van der Voort, L. 2009, ApJ, 694, L128

Leenaarts, J., Rutten, R. J., Reardon, K., Carlsson, M., \& Hansteen, V. 2010, ApJ, 709, 1362

Linsky, J. L., \& Avrett, E. H. 1970, PASP, 82, 169

Lites, B. W. 1974, A\&A, 33, 363

Lites, B. W., Rutten, R. J., \& Kalkofen, W. 1993, ApJ, 414, 345

Makita, M. 2003, Publications of the National Astronomical Observatory of Japan, 7, 1

Martinez Pillet, V., Ruiz Cobo, B., \& Vazquez, M. 1990, Sol. Phys., 125, 211

Martínez-Sykora, J., Hansteen, V., DePontieu, B., \& Carlsson, M. 2009, ApJ, 701,1569

Mattig, W. 1971, Sol. Phys., 18, 434

Mattig, W. 1983, Sol. Phys., 87, 187

Narain, U., \& Ulmschneider, P. 1996, Space Sc. Rev., 75, 453

Oranje, B. J. 1983, A\&A, 124, 43

Pasachoff, J. M., Noyes, R. W., \& Beckers, J. M. 1968, Sol. Phys., 5, 131

Pasachoff, J. M., Jacobson, W. A., \& Sterling, A. C. 2009, Sol. Phys., 260, 59

Penn, M. J., \& Jones, H. P. 1996, Sol. Phys., 168, 19

Pietarila, A., Socas-Navarro, H., \& Bogdan, T. 2007, ApJ, 663, 1386

Rezaei, R., Schlichenmaier, R., Beck, C. A. R., Bruls, J. H. M. J., \& Schmidt, W. 2007, A\&A, 466, 1131

Rezaei, R., Bruls, J. H. M. J., Schmidt, W., et al. 2008, A\&A, 484, 503

Rompolt, B. 1975, Sol. Phys., 41, 329

Rutten, R. J. 2007, in The Physics of Chromospheric Plasmas, ed. P. Heinzel, I. Dorotovič, \& R. J. Rutten, ASP Conf. Ser., 368, 27

Rutten, R. J. 2010, in Recent Advances in Spectroscopy: Theoretical, Astrophysical and Experimental Perspectives, ed. R. K. Chaudhuri, M. V. Mekkaden, A. V. Raveendran, \& A. Satya Narayanan (Springer, Berlin), 163 Rutten, R. J., \& Stencel, R. E. 1980, A\&AS, 39, 415

Sánchez-Andrade Nuño, B., Centeno, R., Puschmann, K. G., et al. 2007, A\&A, 472, L51

Schmidt, W., Knoelker, M., \& Westendrop Plaza, C. 1994, A\&A, 287, 229

Schröter, E. H., Soltau, D., \& Wiehr, E. 1985, Vis. Astron., 28, 519

Shoji, M., Nishikawa, T., Kitai, R., \& Ueno, S. 2010, PASJ, 62, 927

Sivaraman, K. R., Singh, J., Bagare, S. P., \& Gupta, S. S. 1987, ApJ, 313, 456

Sivaraman, K. R., Gupta, S. S., \& Kariyappa, R. 1996, Sol. Phys., 163, 93

Socas-Navarro, H. 2005, ApJ, 633, L57

Socas-Navarro, H., \& Elmore, D. 2005, ApJ, 619, L195

Stencel, R. E. 2009, in The Biggest, Baddest, Coolest Stars, ed. D. G. Luttermoser, B. J. Smith, \& R. E. Stencel, ASP Conf. Ser., 412, 251

Suematsu, Y., Ichimoto, K., Katsukawa, Y., et al. 2008, in First Results From Hinode, ed. S. A. Matthews, J. M. Davis, \& L. K. Harra, ASP Conf. Ser., 397, 27

Teplitskaja, R. B., \& Efendieva, S. A. 1973, Sol. Phys., 28, 369

Vernazza, J. E., Avrett, E. H., \& Loeser, R. 1981, ApJS, 45, 635

von der Lühe, O., Soltau, D., Berkefeld, T., \& Schelenz, T. 2003, in Innovative Telescopes and Instrumentation for Solar Astrophysics, ed. S. Keil, \& S. Avakyan, Proc. SPIE, 4853, 187

Watanabe, T., \& Steenbock, W. 1986, A\&A, 165, 163

Wedemeyer, S., Freytag, B., Steffen, M., Ludwig, H.-G., \& Holweger, H. 2004, A\&A, 414, 1121

White, O. R. 1963, ApJ, 138, 1316

Wilson, O. C., \& Vainu Bappu, M. K. 1957, ApJ, 125, 661

Zaqarashvili, T. V., \& Skhirtladze, N. 2008, ApJ, 683, L91

Zaqarashvili, T. V., Kukhianidze, V., \& Khodachenko, M. 2010, MNRAS, 404, L74

Zirker, J. B. 1962, ApJ, 136, 250

Zirker, J. B. 1968, Sol. Phys., 3, 164

Zwaan, C. 1965, Recherches Astronomiques de l'Observatoire d'Utrecht, 17

Pages 11 to 13 are available in the electronic edition of the journal at http://www . aanda. org 

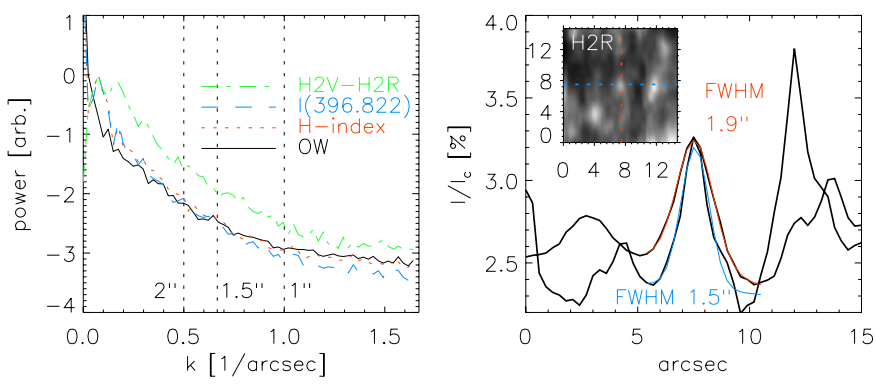

Fig. A.1. Spatial resolution. Left: Fourier power as function of spatial frequency. Right: vertical and horizontal cuts through the center of the $\mathrm{H}_{2 \mathrm{R}}$ map shown as inset at the upper left. Gaussian fits to the central brightening are overplotted with red and blue lines, respectively.

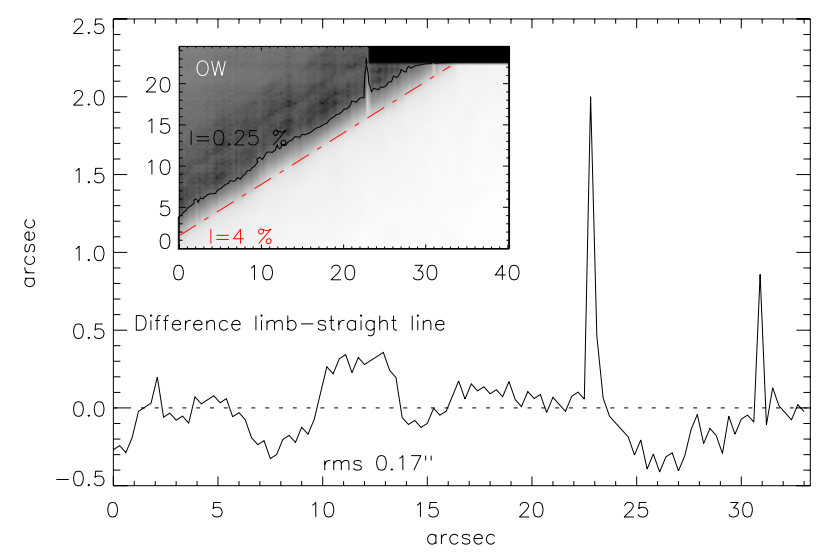

Fig. A.2. Position of the limb in the OW map (inset at the upper left in logarithmic scaling). The inclined dash-dotted line in the inset denotes a linear fit to the limb position. The black line denotes an intensity level of $0.25 \%$. The line plot below the inset shows the difference between the dash-dotted line and the actual limb position in arcsec.

\section{Appendix A: Spatial resolution and residual image motion}

Because observations near the solar limb are usually difficult, especially when one tries to maintain the AO real-time correction, we investigate the achieved spatial resolution. Figure A.1 shows two estimates of the spatial resolution, from the Fourier power as function of the spatial frequency (left) and the extension of an isolated brightening in the $\mathrm{H}_{2 \mathrm{R}}$ map (right). The spatial sampling provides a maximal resolution of $0{ }^{\prime} 6$. The Fourier power levels off to a constant value that is indicative of the noise level between $1^{\prime \prime}$ and $1.5^{\prime \prime}$. The shape of the curves for different wavelength windows (OW, H-index, fixed wavelength at $396.822 \mathrm{~nm}$ ) or the difference between $\mathrm{H}_{2 \mathrm{~V}}$ and $\mathrm{H}_{2 \mathrm{R}}$ is fairly similar, with slightly enhanced power for the intensity difference measure. A Gaussian fit to the extension of the isolated brightening shown as inset in the right panel yielded a $F W H M$ of $1.5^{\prime \prime}$ and $1.9^{\prime \prime}$ on cuts in the horizontal and vertical direction, respectively, with the caveat that the feature presumably will also have an intrinsic spatial extent. The spatial resolution should accordingly be about $1.5^{\prime \prime}$ or slightly better.

To determine the residual image motion caused by the variable seeing and uncompensated image shifts, we measured the

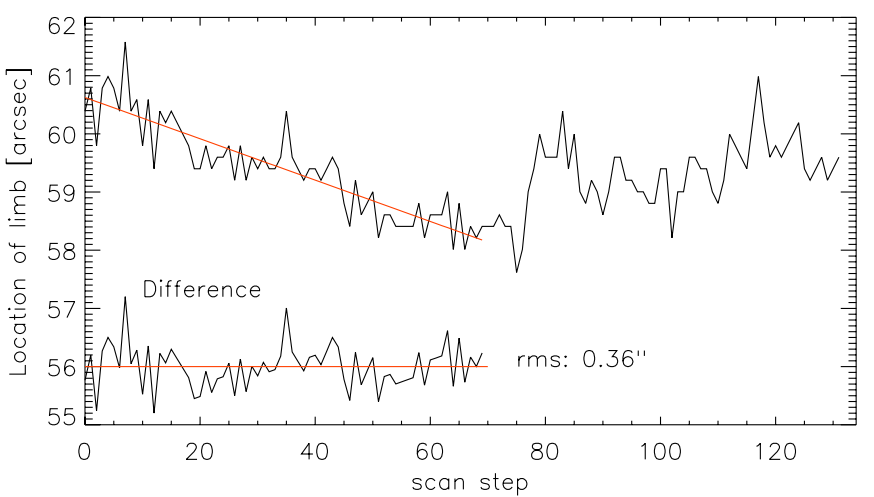

Fig. A.3. Location of the limb for one spatial position in the SJ images. The lower part shows the difference between the red straight line and the limb position.

location of the "white-light" limb in the OW map (inset in Fig. A.2). The limb was defined as the point where the intensity dropped below $4 \%$. We fitted a straight line to the limb location and then derived the difference to the actual limb position at one scan step and the straight line (Fig. A.2). The rms variation of the difference is about 0 ! $^{\prime} 17$. Because this is now derived from the spectra after the 13-s integration, it only provides a temporally averaged value of the image motion. We therefore used the individual slit-jaw (SJ) images for a cross-check. The SJ images are taken at the beginning of each scan step with an exposure time below $20 \mathrm{~ms}$; they are thus not influenced by any temporal averaging. We removed the image motion caused by the spatial scanning perpendicular to the slit from the SJ images, and then determined the location of the limb in the individual images, defined again as the position where the intensity drops below $4 \%$. Figure A.3 shows the distance between the limb and the edge of the SJ image for one randomly chosen image row. In addition to a linear trend that could be caused by a slow drift of the AO lock point or a small tilt angle between the POLIS scan mirror and the slit, the limb position in the SJ images fluctuated with an rms amplitude of about 0.36 . The fluctuations of the atmospheric scattering were below a level of $0.25 \%$ in the OW map, the map is still fairly homogeneous at this intensity level (black line in the inset of in Fig. A.2), with the distance to the limb being the dominating factor for the residual light level.

\section{Appendix B: Additional example spectra}

Figure B.1 shows an example of the multi-lobed profiles found on the disk near a patch of enhanced emission and strong photospheric polarization signal. Figure B.2 shows a series of individual neighboring spectra at a limb distance of $2.5 \mathrm{Mm}$. The Doppler shift of the absorption core relative to the emission peaks reverses from blue to red shift over a spatial scale of about 1.6". The spectra along the slit (Fig. B.3) show the same patterns as those along cuts perpendicular to the limb: a variation of Doppler shifts both in the horizontal direction from scan step to scan step as well as a variation in single cuts along the vertical axis. Figures B.4 and B.5 show the individual profiles of cut no. 2 and the scan step no. 66. In the first case, the distance given at the left of each profile is measured perpendicular to the limb, in the latter case it only reflects the location along the slit. 


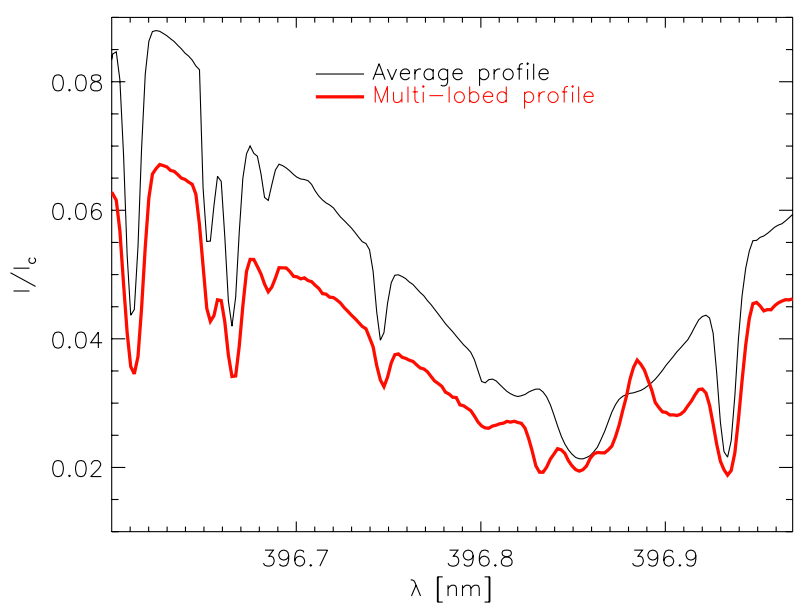

Fig. B.1. Ca profile with more than two reversals (thick red). The thin black line shows the average profile for comparison.
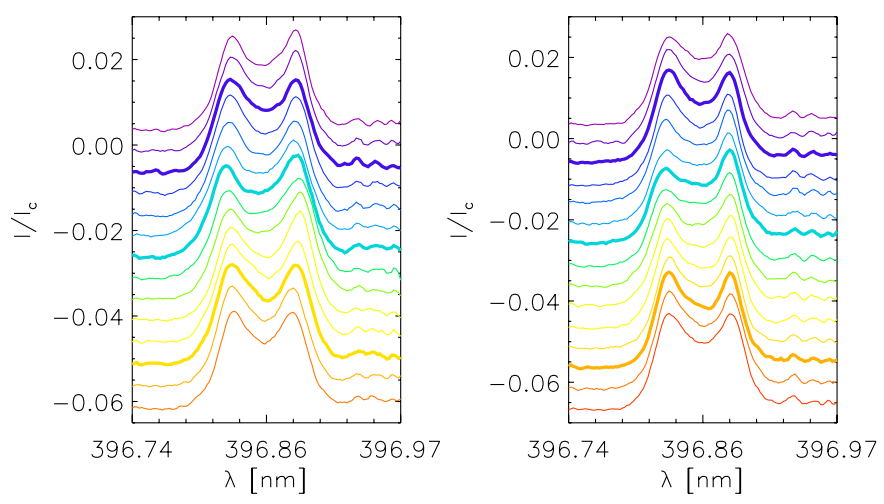

Fig. B.2. Individual spectra at a limb distance of $2.5 \mathrm{Mm}$. Left: spectra from $x=4.5^{\prime \prime}$ to $8.4^{\prime \prime}$. Thick lines indicate profiles with large shifts. Right: same from $x=9.3^{\prime \prime}$ to $13.5^{\prime \prime}$. The position values refer to the spatial scale given in Fig. 7.

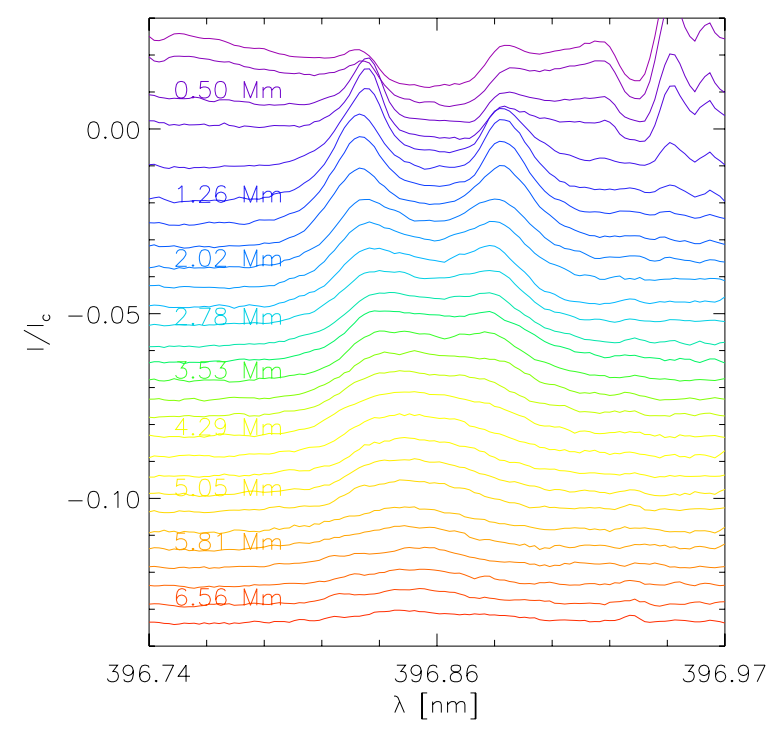

Fig. B.4. Spectra of cut no. 2 (see Fig. 3) with increasing limb distance. The limb is at the top, each third profile is labeled with its corresponding height.

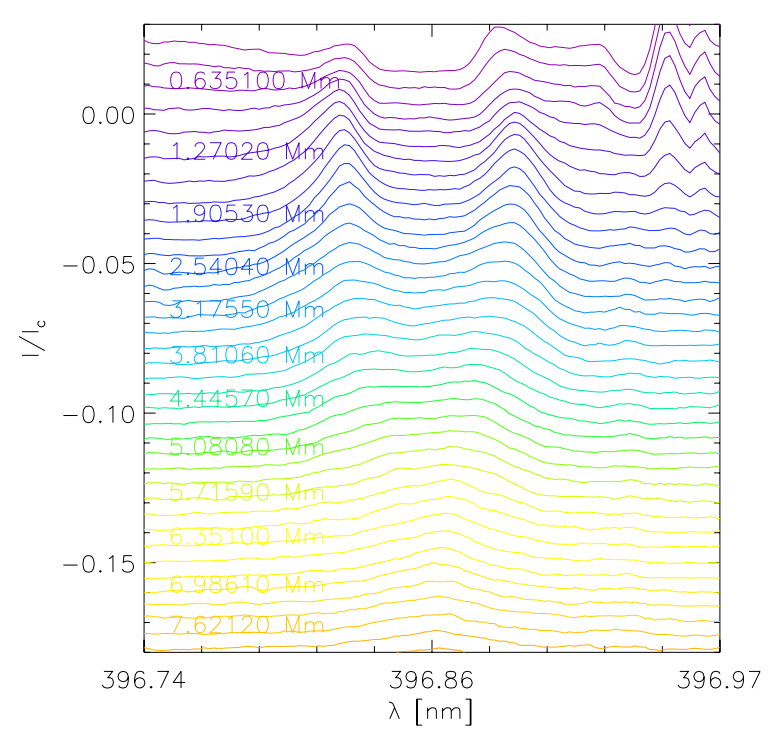

Fig. B.5. Spectra of scan step no. 66 (see Fig. 3) with increasing position along the slit. The limb is at the top, each third profile is labeled with its corresponding height. 
C. A. R. Beck and R. Rezaei: Spectroscopy at the solar limb
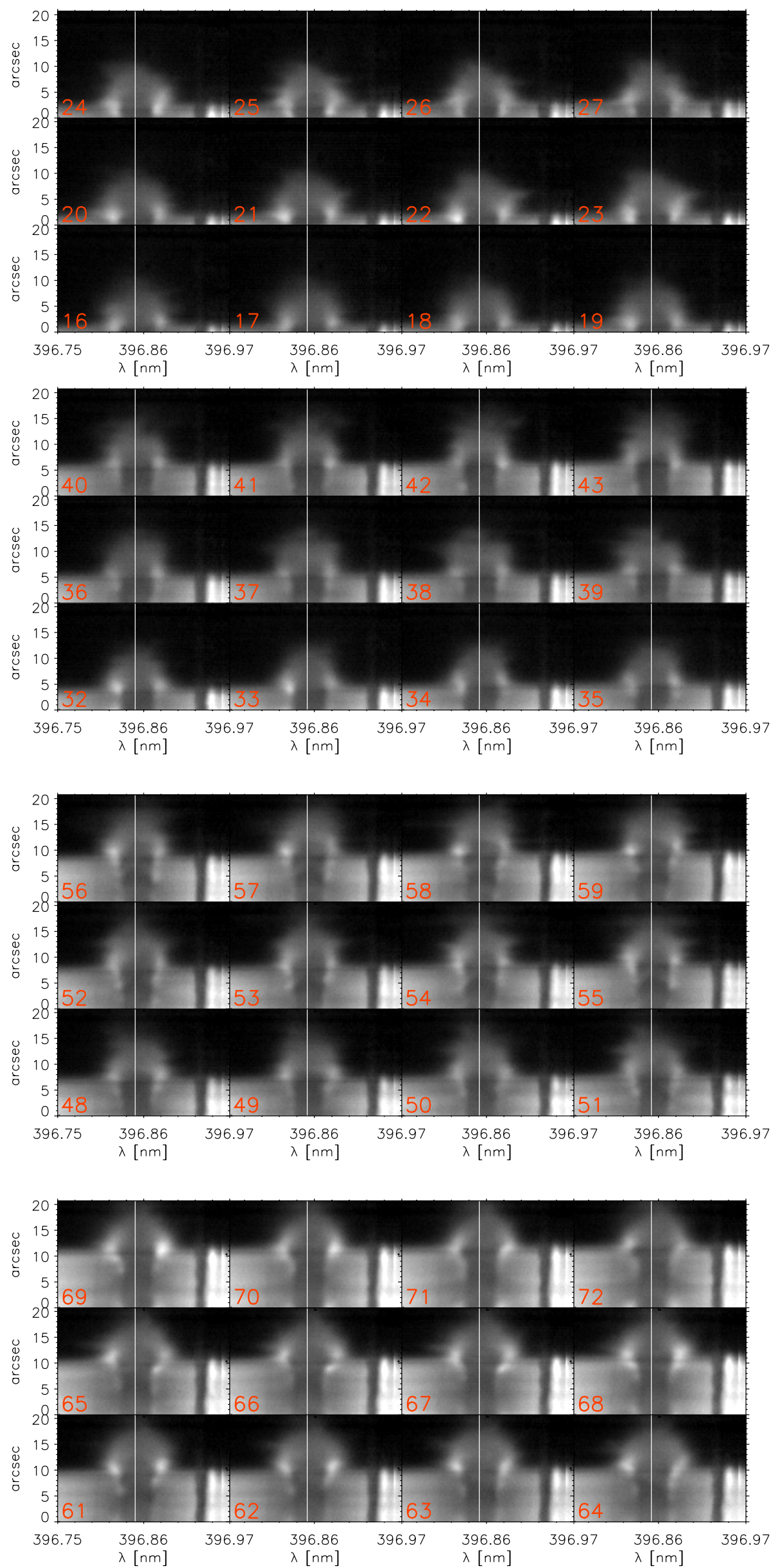

Fig. B.3. Spectra along the slit around the scan step 21 (top panel), step 37 (second panel), step 53 (third panel), and step 66 (bottom panel). The scan step of each cut along the slit is given at the lower left of each panel, increasing from left to right and from bottom up. The $y$-axis gives the distance along the cut, the limb is thus located at varying positions. The vertical white line denotes the rest wavelength of $\mathrm{Ca}$ II $\mathrm{H}$. 